a single vortex flow structure, four showed multiple vortices and one had a helical flow pattern without a clear vortex structure. A strong positive correlation exists between the flow $\mathrm{KE}$ and enstrophy density.

Conclusions and Implications This study suggests that flow energy in the RA is mainly rotational, part of which is convected by the highly helical SVC and IVC inflows. Multiple vortices tend to be associated with higher dissipation rates in the central RA region due to turbulence. The rotational nature of the flow in the RA maintains KE better than non-rotational flow. RA flow characteristics are highly related to the helicity content in the caval veins, as well as the KE flux intensity. Lower caval helicity or IVC KE flux dominance tends to favour single vortex formation while the opposite tends to lead to multiple vortices or the rare helical flow patterns. Atria lacking single vortex flow are inclined to have a larger energy input from atrial contraction.

Conflict of Interest $\mathrm{n} / \mathrm{a}$

\section{BS7 PLASMA DESMOSINE AS A BIOMARKER IN ACUTE AORTIC SYNDROME}

${ }^{1}$ Maaz Syed, ${ }^{2}$ Zaid Iskander, ${ }^{3}$ Alexander Fletcher, Samuel Debono, ${ }^{3}$ Marc Dweck, ${ }^{4}$ Jeffrey T J Huang, ${ }^{5}$ Calvin Chin, ${ }^{3}$ David Newby, ${ }^{2}$ Anna Maria Choy. ${ }^{1}$ BHF Department for Cardiovascular Sciences, University of Edinburgh, Edinburgh, UK; ${ }^{2}$ Division of Molecular and Clinical Medicine, University of Dundee; ${ }^{3}$ Centre for Cardiovascular Sciences, University of Edinburgh; ${ }^{4}$ School of Medicine, University of Dundee; ${ }^{5}$ National Heart Centre Singapore

10.1136/heartjnl-2021-BCS.205

Introduction Acute aortic syndromes (AAS) include aortic dissection, intramural haematoma and penetrating aortic ulcer, all of which are caused by aortic wall failure and associated with significant mortality. Although, treatment options currently include early surgical intervention or aggressive medical management, disease progression and devastating complications remain commonplace. Early diagnosis of AAS as well as ability to predict those at the highest risk of disease progression would represent significant progress in the care these patients receive. Desmosine is the cross-link component in the elastin molecule and is exclusively released from mature elastin breakdown, thus is a physiologically relevant biomarker of aortic elastin degradation. The aim of the study was to test the hypothesis that plasma desmosine (pDES) concentrations are elevated in AAS and has prognostic value in indentifying those at risk of significant disease progression.

Method Patients over 25 years old with radiologically confirmed acute aortic syndrome were recruited as part of a prospective observational study (NCT03647566). Demographic details, AAS sub-category, time from index acute aortic syndrome event and pDES concentrations measured by stable isotope dilution LC-MS/MS were recorded at baseline. Baseline and follow up maximal aortic diameters were measured on contrast-enhanced computed tomography (CT) and change in aortic diameter over time was calculated. Control plasma desmosine samples were obtained at a 2:1 ratio control subjects participating in the United Kingdom Aneurysm Growth Study. Data presented as mean \pm standard deviation or median [interquartile range].

Results Plasma desmosine concentrations were measured in 53 patients (64 [53 to 71] years) with acute aortic syndromes and 106 control subjects (53 [44 to 60] years). In patients with AAS, pDES concentrations were almost twice those of control subjects $(0.58 \pm 0.26$ vs $0.27 \pm 0.07, \mathrm{p}<0.001)$. In those with AAS, plasma desmosine concentrations were seen to be highest at presentation, and reduced over time from the aortic syndrome event $(\mathrm{R}=0.51, \mathrm{p}=0.003)$. Plasma desmosine concentration was the only variable associated with increasing aortic diameter over time $(\mathrm{R}=0.34, \mathrm{p}=0.014)$.

Conclusion Plasma desmosine concentrations are elevated in patients with AAS, peak at the time of presentation and represents a promising biomarker for early identification and risk stratification in patients with AAS.

Conflict of Interest none to declare

\section{BS8 GENETICALLY-DETERMINED SERUM CALCIUM LEVELS INFLUENCE MARKERS OF VENTRICULAR REPOLARISATION: A MENDELIAN RANDOMISATION STUDY}

${ }^{1}$ William Young, ${ }^{1}$ Helen Warren, ${ }^{2}$ Dennis Mook-Kanamori, ${ }^{1} J u l i a$ Ramirez, 'Stefan van Duijvenboden, ${ }^{3}$ Michele Orini, ${ }^{1}$ Andrew Tinker, ${ }^{2}$ Diana van Heemst, ${ }^{3}$ Pier Lambiase, ${ }^{2} \mathrm{~J}$ Wouter Jukema, ${ }^{1}$ Patricia Munroe, ${ }^{2}$ Raymond Noordam. ${ }^{1}$ Queen Mary University of London, London, UK; ${ }^{2}$ Leiden University Medical Center; ${ }^{3}$ University College London

\subsection{6/heartjnl-2021-BCS.206}

Introduction Electrocardiographic (ECG) markers of ventricular depolarisation and repolarisation are associated with an increased risk of arrhythmia and sudden cardiac death. Our prior work indicated lower serum calcium concentrations are associated with longer QT and JT intervals in the general population. Here, we investigate whether serum calcium is a causal risk factor for changes in ECG measures using Mendelian Randomisation (MR).

Methods We performed a new genome-wide association study (GWAS) for serum calcium in $>300,000$ European-ancestry participants from UK-Biobank. Independent lead variants were extracted to be used as instrumental variables (figure 1). Twosample MR analyses were performed to approximate the causal effect of serum calcium on QT, JT and QRS intervals using an inverse-weighted method in 76,226 UK-Biobank participants with ECG data, not contributing to the serum calcium GWAS. A secondary analysis wase performed using lead variants from a calcium GWAS corrected for serum albumin. Sensitivity analyses were performed using contemporary methods to test for the presence of horizontal pleiotropy.

Results 205 independent lead calcium-associated variants were used as instrumental variables for Mendelian Randomisation. A decrease of $0.1 \mathrm{mmol} / \mathrm{L}$ genetically-determined serum calcium, was associated with longer QT $(3.01 \mathrm{~ms}$ (95\% CI 2.03, $3.99))$ and JT $(2.89 \mathrm{~ms}(1.91,3.87))$ intervals. A weak association was observed for QRS duration in secondary analyses only $(0.39 \mathrm{~ms}(0.08,0.69))$. Results were concordant in all sensitivity analyses.

Conclusion These analyses support a causal effect of serum calcium levels on ventricular repolarisation, in a middle-aged population of European-ancestry where serum calcium concentrations are likely stable and chronic. Modulation of calcium concentration may therefore directly influence cardiovascular disease risk. Further research into the effects of serum calcium concentration on arrhythmogenesis is warranted and calcium variants could be considered for inclusion in genetic risk score models for predictive testing. 


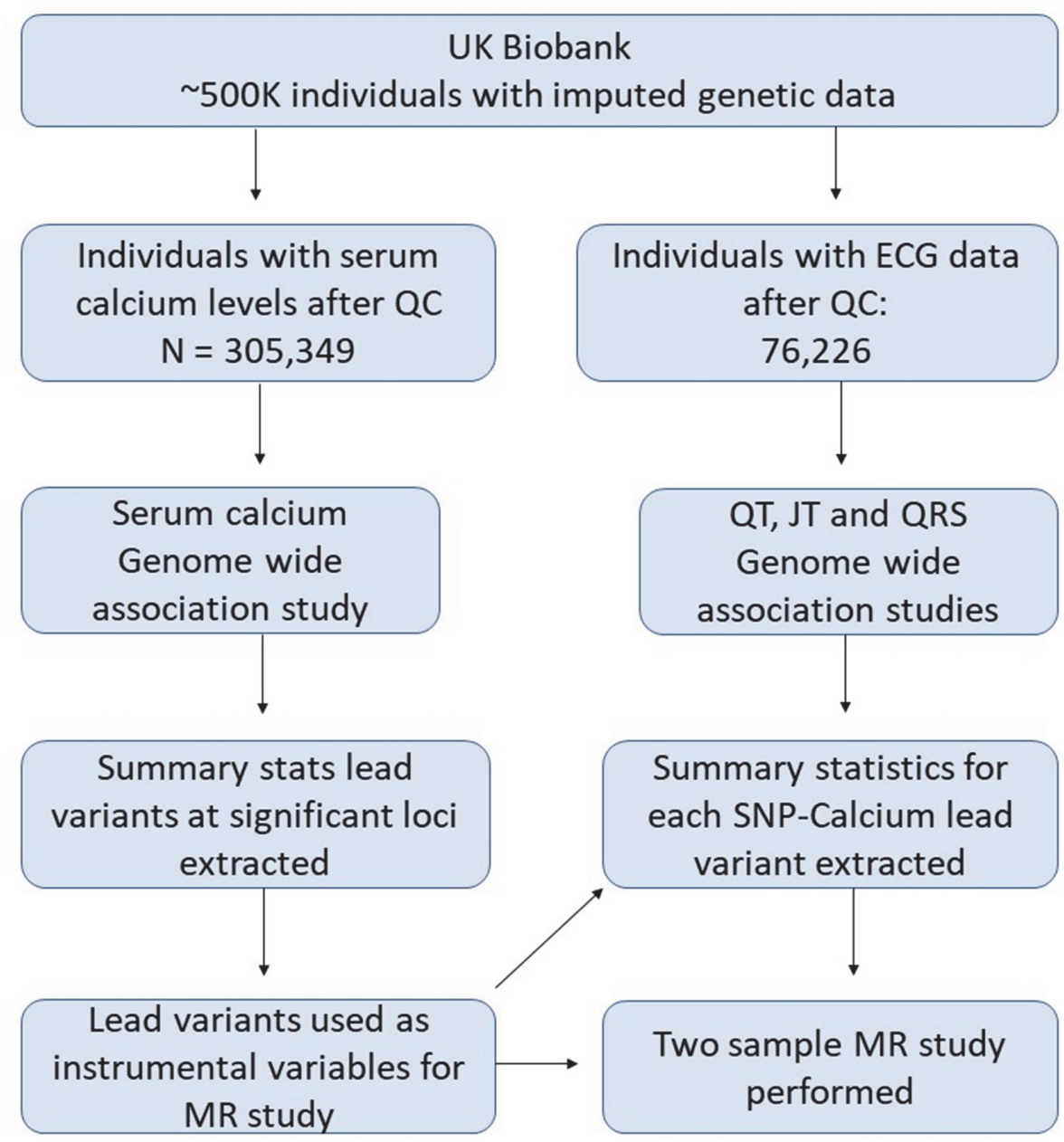

\section{Abstract BS8 Figure 1}

Conflict of Interest None

\section{BS9 REGIONAL ALTERATIONS TO THE TRANSVERSE-TUBULE NETWORK IN AN OVINE MODEL OF MYOCARDIAL INFARCTION}

Tharushi Perera, Christian Pinali, Emma Raddliffe, Barbara Niort, Charlene Pius, Andrew Trafford. University of Manchester, Manchester, UK

\subsection{6/heartjnl-2021-BCS.207}

The highly organised transverse tubule (t-tubule) network consisting of invaginations of the cell sarcolemma facilitates synchronous cardiac myocyte contraction. This study aimed to investigate post-myocardial infarction (MI) t-tubule remodelling in infarct border and remote regions in a translationally relevant ischaemia reperfusion injury MI model.

Six adult sheep were used in this study $(n=3$ MI, $n=3$ control). Eight weeks after MI, left ventricular tissue was collected from the remote and border MI regions and from control sheep, processed and imaged using 3D scanning electron microscopy. The t-tubule network was manually segmented using 3dmod. One-way ANOVA with Tukey's post-hoc correction, unpaired t-tests or Mann-Whitney $U$ test were used where appropriate.
Marked disorganisation of the t-tubule network was observed in the border region following MI. Quantitative analysis revealed that in comparison to the control sheep myocardium, the MI border zone had a decreased t-tubule count $(0.07 \pm 0.007$ tubules per $\mu \mathrm{m} 3$ in control vs $0.05 \pm 0.004$ tubules per $\mu \mathrm{m} 3$ in border; $\mathrm{p}=0.02$ ) and showed t-tubule dilation $(405 \pm 22 \mathrm{~nm}$ in control vs $533 \pm 30 \mathrm{~nm}$ in border; $\mathrm{p}=0.02$ ). Whilst there was minimal disorganisation and loss of t-tubules in the MI remote region, we observed increased t-tubule length as a fraction of the cell diameter $(0.41 \pm 0.04$ in control vs $0.56 \pm 0.04$ in remote; $p=0.045)$. In addition to gross t-tubule remodelling, we also noted post-MI fragmentation of t-tubules, particularly in the border region. In comparison to control, the number of t-tubule fragments per $\mu \mathrm{m} 3$ was increased in the post MI heart (control, $0.17 \pm 0.1$ fragments per $\mu \mathrm{m} 3$; border, $2.21 \pm 0.7$ fragments per $\mu \mathrm{m} 3$; remote, $1.20 \pm 0.4$ fragments per $\mu \mathrm{m} 3 ; \mathrm{p}=0.04$ border vs control; $\mathrm{p}=0.02$ remote vs control). The volume occupied by fragments as a percentage of the cell volume was also higher following MI (control, $0.003 \pm 0.002 \%$; border, $0.071 \pm 0.023 \%$; remote $0.014 \pm 0.005 \%$; $=0.003$ border vs control; $\mathrm{p}=0.013$ border vs remote). Whilst there was no difference in fragments density between the remote and border regions, there was an increase in the volume of cell occupied by fragments in the MI border region compared to remote. This is explained by a larger average fragment 